\title{
Lateral column lengthening osteotomy in idiopathic adolescent flatfoot A systematic review
}

\author{
S.A.Khaleel, A.S.ElGazar, H.E.Farag and A.M.Fahmy \\ Orthopedics \& surgery, Dept., Faculty of Medicine, Benha Univ., Benha, Egypt \\ E-Mail:Arafa289@gmail.com
}

\begin{abstract}
Although research is abundant and the biomechanical effects of various treatments on the hindfoot are well detailed, there is no clear agreement about the optimum technique or procedure to conduct in the case of flexible pes planovalgus foot deformity. This is a systematic evaluation to assess the impact on the existing published literature of idiopathic flatfoot lateral column lengthening osteotomy in teenagers. This review was conducted according to the 2015 PRISMA-P checklist (Preferred reporting items for systematic review and meta-analysis protocols). Twenty-one articles were evaluated against inclusion criteria by reading the entire text and seven papers were found. The mean AOFAS scores were 56.08 (range 23-78) while the mean AOFAS postoperative scores were 92.21 (range 34-100). Two studies that utilised criteria showed that Good/Excellent resulted in 72 percent (23/32 feet) and Fair/Poor, 18 percent (9/32) feet. Subjective and objective scoring were utilised for ACFAS, however only postoperative results were provided. Complications in $27(16.5 \%)$ of 164 individuals have been recorded. Sural neuropathy, pseudoarthrosis, non-union, calcaneocubosis, metal irritation, and adhesion were frequent consequences. Child and young adults with lateral column lengthening surgical treatments for Pes planovalgus deformity had excellent, medium-term, clinical and radiological outcomes with high patient satisfaction and an acceptable degree of complication.
\end{abstract}

Keywords: Lateral column, flatfoot.

\section{Introduction}

Idiopathic pes planus in teenagers and young adults is a frequent foot condition [1].Pes planovalgus is the most frequent foot defect in children and has a broad variety of severity and symptoms. Surgery is usually recommended for severe malalignment, which is resistant to surgery. Lateral column extension is regarded to be an attractive alternative because it does not require arthrodesis and allows for continued growth and development [2]. Angles and other radiographic characteristics in children and teenagers may be utilised to create a radiographic categorization system that offers helpful treatment advice in clinical practise when examining both the dorsoplantable and the side views. [3]

Porous titanium LCL showed reduced nonunion rates, better X-rays and pain alleviation. [4].Adult flatfoot deformity acquired is defined by the abduction and collapse of the medial length arch. Lateral column extension osteotomies mainly address the deformity of the abduction [5]. An increase in lateral plantar pressure was predictive of correction of abduction deformity [5] Lengthening of the side column by osteotomy with the help of the Evans is frequently utilised for adult and paediatric flatfoot repair. However, certain studies showed that the osteotomy of Evans is associated with higher calcaneocuboid joint pressures and an increased risk of joint arthritis [6] In some measure, the lateral column lengthening decreases the calcaneocube joint pressure with the flatfoot deformity. The $8 \mathrm{~mm}$ lengthening treatment may decrease the incidence of subsequent calcaneocuboid arthritis [6] Despite plenty of research that details the biomechanical impact on the hindfoot of various operating techniques, there is no clear agreement as to whether the method or treatments for flexible pes planovalgus foot deformity are best performed. There is thus no one answer to this issue; the surgeon must treat each patient individually and choose the technique that works best for whatever foot pathology they have 7 [7].

This is a systematic evaluation to assess the impact on the existing published literature of idiopathic flatfoot lateral column lengthening osteotomy in teenagers.

\section{Patient and method}

This review was performed in line with PRISMAP 2015 checklist (Preferred reporting items for systematic review and meta-analysis protocols). The Pubmed, Cochrane, CINAHL, EMBASE, Google Scholar and bibliography of articles was searched for all relevant articles against search criteria. The keywords used for search are summarised in Table (1). The authors conducted literature searches using keywords and abstracts for the electronic databases. Authors evaluated the papers fulfilling

-joint preservation operations such as arthrodesis.

We gathered demographic data of patients, including number of operated patients and foot, patient age, follow-up time. Documented clinical and radiological results. We utilised talo-metatarsal, tano-navicular angle coverage, and calcaneal angle for radiological measures, as other studies have used. Furthermore, we documented pathology, extra procedures, postoperative treatment, problems and reoperations. The quantity and type of subsequent soft tissue operations were also recorded.

\section{Statistical analysis}

Review manager was used to conduct this review and differences with $\mathrm{p}$-value $<0.05$ was considered statistically significant. An independent sample t- test was performed to analyse preoperative and postoperative radiological measurements of calcaneal 
pitch angle, lateral talo-metatarsal angle and talonavicular coverage angle.

Observations and results

The initial search of the literature with the above keywords identified 391 articles. We excluded 286 articles with their titles including duplicates and identified 105 for further evaluation of abstract. Twenty-one studies were assessed by reading full text against inclusion criteria and identified seven studies Table (2).

There were 237 calcaneal lengthening osteotomies performed on 164 patients in these nine studies. The mean age at the time of surgery was 14.83 years. (Range 5.7-42) and mean length of follow up was 61.2 Months (Range 15.9-156). There were 33 females and 29 males of the 62 patients in four studies where information available. Table [3] has demographic information.

Table (1) Keyword searches for identifying articles.

\section{Results}

\subsection{Radiological outcome}

Pes planovalgus is complex deformity involving multiple joints and in three dimensions. There were numerous radiological parameters reported and available in four of the seven studies. Calcaneal pitch angle, lateral talo-first-metatarsal angle, AP Talonavicular coverage angle were most commonly used in the studies therefore were included in the review. From these publications as listed above, mean preoperative calcaneal pitch angle was 10.5 (SD 3.3, Range 4.4-12.3) and the mean postoperative calcaneal pitch Angle was 21.5 (SD 4.9, Range 17.3-31) (p Value $=0.001)$. The mean preoperative Lateral Talo$1^{\text {st }}$ metatarsal angle was 18.44 (SD 5.78 Range 15.527.5) and improved to 6.69 (SD 6.4 Range 0.3-13.7) $(\mathrm{p}$ Value $=0.001)$

\begin{tabular}{llc}
\hline Planovalgus surgery & AND & Children \\
\hline Planovalgus surgery & AND & Paediatric \\
Planovalgus surgery & AND & Adolescent \\
Planovalgus surgery & AND & Paediatric \\
Lateral column lengthening & AND & Children \\
Lateral column lengthening & AND & Paediatric \\
Lateral column lengthening & AND & Adolescent \\
Calcaneal lengthening osteotomy & AND & Children \\
Calcaneal lengthening osteotomy & AND & Paediatric \\
Calcaneal lengthening osteotomy & AND & Adolescent \\
Calcaneal osteotomy & AND & Juvenile \\
Pes Planovalgus surgery & AND & Paediatric \\
Flatfoot surgery & AND & Paediatric \\
\hline
\end{tabular}

Table (2) Flow Diagram of the study.

\begin{tabular}{|c|}
\hline $\begin{array}{c}\text { Initial search with key words } \\
1975-2019 \\
\mathbf{4 3 6} \text { citations }\end{array}$ \\
\hline 150 Non Duplicae citations screened \\
\hline 127 Articles Excluded After title/abstract screening \\
\hline 23 Articles retrieved \\
\hline 14 Articles Excluded After full text screening \\
\hline 9 Articles Included \\
\hline
\end{tabular}


Table (3) Demographic information and postoperative management (NWB: Non weight bearing, WB: Weight bearing, SL Cast: Short Leg Cast).

\begin{tabular}{|c|c|c|c|c|c|}
\hline References & $\begin{array}{c}\text { No of } \\
\text { patients }\end{array}$ & $\begin{array}{c}\text { No of } \\
\text { feet }\end{array}$ & $\begin{array}{c}\text { Follow-up in } \\
\text { months }\end{array}$ & $\begin{array}{l}\text { Patient age } \\
\text { (Range) }\end{array}$ & Post-op plan \\
\hline $\begin{array}{l}\text { Matthews et } \\
\text { al., }[8]\end{array}$ & 34 & 43 & 43.50 & $27.3(9-62)$ & $\mathrm{NWB}+$ Cast \\
\hline $\begin{array}{l}\text { Marengo et } \\
\text { al., }\end{array}$ & 27 & 38 & 15.9 & $13.3(7.8-17)$ & $\begin{array}{l}\text { V Vulpius lengthening, PB peroneus } \\
\text { brevis lengthening, }\end{array}$ \\
\hline $\begin{array}{l}\text { Phillips et } \\
\text { al. }{ }^{[10]}\end{array}$ & 20 & 23 & 156 & $15(9-42)$ & Cast 16/52, WB $4 / 52$ \\
\hline $\begin{array}{l}\text { Akimau et } \\
\text { al. }\end{array}$ & 12 & 20 & 54 & $12.6(5.7-16.3)$ & NWB 6/52, Insole $3 / 12$ \\
\hline $\begin{array}{l}\text { Anderson et } \\
\text { al. }{ }^{[12]}\end{array}$ & 5 & 9 & 80 & $10.4(6-13)$ & Specified SL cast 2 cases \\
\hline Oh et al. ${ }^{[13]}$ & 10 & 16 & 62 & $15.6(10-22)$ & Cast $8-10 / 52$ NWB \\
\hline Viegas et al. ${ }^{[14]}$ & 17 & 34 & 24.9 & $12.1(8-18)$ & SL Cast 6/52 NWB \\
\hline $\begin{array}{l}\text { Moraleda et } \\
\text { al. }{ }^{[15]}\end{array}$ & 21 & 33 & 63 & $11.6(9.1-15.1)$ & NS \\
\hline $\begin{array}{l}\text { Yontar et } \\
\text { al. }{ }^{[16]}\end{array}$ & 18 & 21 & 51.2 & $15.6(9-25)$ & SL Cast 6/52 NWB \\
\hline Totals & 164 & $\begin{array}{c}237 \\
\text { Range }\end{array}$ & $\begin{array}{c}61.17 \\
15.9-156\end{array}$ & $\begin{array}{c}14.83 \\
(5.7-42)\end{array}$ & *Mean \\
\hline
\end{tabular}

The mean preoperative AP Talo-navicular coverage angle also improved from 28.06 (SD 5.7, Range 21.8-38.6) to 8.83 (SD 8.01, Range 3.4-13.7) ( $\mathrm{p}$ value < 0.0001). We used GraphPad Software to calculate statistical significance using sample t- test.

Normal values are reported variably in the literature. According to Escobedo et $\mathrm{al}^{17}$, normal calcaneal pitch angle is 18-20, however range from 17 to 32 is reported as normal in literature. The talo $-1^{\text {st }}$ metatarsal angle greater than 4 and talo-navicular coverage angle greater than 7 are considered abnormal.

\subsection{Clinical outcome}

Five AOFAS Score publications (129 feet) were used to measure clinical and preoperative outcomes in four investigations. In an article two articles (32 feet) used the author and one utilised the ACFAS Score for clinical result measurement and Mosca clinical score.

The mean AOFAS scores were 56.08 (range 2378 ) while the mean AOFAS postoperative scores were 92.21 (range 34-100). Two studies that utilised criteria showed that Good/Excellent resulted in 72 percent (23/32 feet) and Fair/Poor, 18 percent (9/32) feet. Subjective and objective scoring were utilised for ACFAS, however only postoperative results were provided.

\section{Complications}

Complications in $27(16.5 \%)$ of 164 individuals have been recorded. Sural neuropathy, pseudoarthrosis, non-union, calcaneocubosis, metal irritation, and adhesion were frequent consequences. Moraleda et al.15 reported subluxation of calcaneocubes at 51.5 feet (17/33) after osteotomy of the calcaneal extension but did not alter the result. This article contains no detailed information of the surgical or postoperative regimes. Marengo et al. also revealed postoperative radiographs of 76 percent of the foot with a joint subluxation of calcaneocuboids. There was one instance of postoperative hematoma at the donor site of iliac bone transplant.

In 3 trials, further surgery/recurrence was observed in 7 (12.3 percent) of 57 foot. The most frequent cause cited was recurrence. Another sign was the lysis of adhesions and the liberation of the tarsal tunnel. Further analysis could not be carried out on the basis of available information.

Low problems include transmission of pain in 2 (3.17) patients $(2(3.17)$, cotton site surgery in $1(1.59)$ patient, superficial cellulite in 2 patients, (3.17) and painful hardware in 1 (1.59) patient.

\subsection{Surgical procedure}

Most review studies used the Evans18-described surgical method and adapted Mosca19 with just small changes. Osteotomy proximal to the calcaneocub joint is done during an interval between the anterior and the middle facets of the calcane. The tricortical tricortical crest autograft or allograft shaped trapezoid may be utilised for lengthening. Other changes to the osteotomy site are reported as short-term outcomes case series.

A number of other soft tissue and osseous operations were also used to treat deformities besides calcaneal lengthening, as mentioned above. The soft tissue procedure included a recession of the Achilles tendon or gastrocnemius aponeurosis, brief extension of the peroneus to the longus transfers, accessory marine excision, and post repositioning of the tibial, talo navicular joint of the capsule, including the advance of the post tendon of tibialis. A medial calcaneal slip and a medial cuneiform osteotomy 
comprised more bone operations. All of the interventions covered in the review included the extension of the lateral column and other operations, depending on the success of the repair of the deformity.

Table (3) summarises the post-operative treatment of patients in six trials. Short leg cast was utilised for 6-16 weeks with weight bearing change in most trials. Non-weight bearing was first treated for 3-8 weeks. Long leg cast was utilised for 6 weeks in one research.

\section{Discussion}

The deformity of Pes planovalgus is a common foot defect observed in youngsters. According to Rome et al. [20] there is no evidence for the effectiveness of foot orthosis nor any other nonsurgical procedure for asymptomatic individuals from randomised controlled studies. Optimum orthosis choice and long-term prognosis also remains uncertain in symptomatic individuals. These studies generally suggest that operational surgery is required if extended non-surgical treatment fails to alleviate pain and discomfort. Arthrodesis and joint conservation operational management of Pes planovalgus is reported with short-term outcomes, but medium- to long-term results are uncertain. Lateral column extensions are considered an attractive alternative since they do not require arthrodesis and allow for additional growth and foot development. The objective of this systematic review was to assess the medium and long term clinical and radiological outcome of surgical previously published literature on lateral column lengthening.

This systemic examination includes restrictions on the kind of research covered in the examination. All articles have been retrospective case studies of a limited number of single-unit patients (5-34). These trials contain no control group or randomization and are thus likely to be assigned, selected and randomised.

Patients, surgeons and staff collecting data were aware of the surgical procedure, leading to performance and detection differences. We analysed research for the comprehensiveness of patient information about clinical, radiological results and complications for this systemtic examination. All studies including full radiological information, clinical results and complications for all participants were classified as having a low risk of bias for the purpose of this publication.

The age range and the aetiology of these studies differs, making it difficult for paediatric patients to isolate the outcomes of surgical surgery for pes planovalgus. Results for a specific underlying illness such as idiopathic, congenital or neurogenic were similarly difficult to identify. Most investigations used the extension of the lateral column as reported in Evans18 and modified in Mosca19 with very minor modifications. Variability of the extra operation was done by the surgeon at the time of surgery as considered suitable. Because of the variety of aetiology and information in the trials, it was unable to make strong conclusions about further procedures. There was significant heterogeneity in post-operative studies and reporting data, making it impossible to conclude evidence on usage of a certain kind of regime.

For reporting findings of these investigations, a variety of outcomes measures and radiological parameters have been used. Only 4 research utilised validated results scoring (AOFAS) to provide preoperative results in three trials. AOFAS Score was significantly improved postoperatively. Other research employed various secondary outcomes measures to prevent the combination of the findings. Other studies used patient satisfaction following surgery and the author criteria based on pain alleviation, functional improvement and clinical appearance. Radiological parameters differed between research, however in these studies we analysed three of those most often utilised. These radiological markers have improved significantly in postoperative patients. Reporting recurrence or additional operation were inconsistent and reported in three of the seven trials included in the study. There was variation in the data reporting level in these studies with either a range of radiological parameter or a standard deviation, therefore preventing descriptive statistics from being carried out.

\section{References}

[1] N.S.Yontar, Surgical treatment results for flexible flatfoot in adolescents. Acta Orthopaedica et Traumatologica Turcica.VOL. 50(6), PP. 655-659,2016.

[2] V.Kumar, T.M.Clough, Talar neck fracture-A rare but important complication following subtalar arthroereisis. The Foot.VOL. 24(4), PP. 169-171,2014.

[3] C.Bourdet, Flatfoot in children and adolescents. Analysis of imaging findings and therapeutic implications. Orthopaedics \& Traumatology: Surgery \& Research.VOL. 99(1), PP. 80$87,2013$.

[4] C.E.Gross, Radiographic Outcomes Following Lateral Column Lengthening With a Porous Titanium Wedge. Foot \& Ankle International.VOL.36(8),PP. 953-960,2015.

[5] J.R.Baxter, Graft Shape Affects Midfoot Correction and Forefoot Loading Mechanics in Lateral Column Lengthening Osteotomies. Foot \& Ankle International.VOL. 35(11),PP. 11921199,2014.

[6] J.Xia, Biomechanical Analysis of the Calcaneocuboid Joint Pressure After Sequential Lengthening of the Lateral Column. Foot \& Ankle International.VOL. 34(2),PP. 261266,2013.

[7] A.J.Roche, J.D.F.Calder, Lateral Column Lengthening Osteotomies. Foot and Ankle Clinics.VOL. 17(2),PP. 259-270,2012. 
[8] M.Matthews, Long-Term Outcomes of Corrective Osteotomies Using Porous Titanium Wedges for Flexible Flatfoot Deformity Correction. J Foot Ankle Surg.VOL.57(5),PP. 924-930,2018.

[9] L.Marengo, Clinical and radiological outcome of calcaneal lengthening osteotomy for flatfoot deformity in skeletally immature patients. Eur J Orthop Surg Traumatol.VOL. 27(7),PP. 989996,2017.

[10] G.E. Phillips, A review of elongation of os calcis for flat feet. J Bone Joint Surg.VOL.65 (1),PP.15-18,1983.

[11]P.Akimau, M.Flowers, Medium term outcomes of planovalgus foot correction in children using a lateral column lengthening approach with additional procedures 'a la carte. Foot Ankle Surg.VOL.20,PP.26-29,2014.

[12] A.F.Anderson, S.Benjamin Fowler ,Anterior calcaneal osteotomy for symptomatic juvenile pes planus. Foot Ankle.VOL.4(5),PP.13-15 ,1984.

[13]I.Oh, B.R.Williams, S.J.Ellis, D.J.Kwon, J.T.Deland, Reconstruction of the symptomatic idiopathic flatfoot in adolescents and young adults. Foot Ankle Int.VOL.32(3),PP.20-32, 2011

[14] G.V.Viegas, Reconstruction of the pediatric flexible planovalgus foot by using an evans calcaneal osteotomy and augmentative medial split tibialis anterior tendon transfer. J Foot Ankle Surg.VOL. 42(4),PP.50-14,2003.

[15]L.Moraleda, M.Salcedo, T.P.Bastrom, D.R.Wenger, J.Albiñana, S.J.Mubarak, Comparison of the calcaneo-cuboid-cuneiform osteotomies and the calcaneal lengthening osteotomy in the surgical treatment of symptomatic flexible flatfoot. J Pediatr Orthop.VOL. 32(8),PP.821-829,2012.

[16] N.S.Yontar, T.Ogut, M.F.Guven, H.Botanlioglu, G.Kaynak, A.Can, Surgical treatment results for flexible flatfoot in adolescents. Acta Orthop Traumatol Turc.VOL.50,PP.655-659,2016.

[17] M.Escobedo Eva, J .Pinney Stephen, C .Hunter John, J.Sangeorzan Bruce ,Evaluation of Adult Foot Alignment.

[18]D.Evans, Calcaneo-valgus deformity. J Bone Joint Surg.VOL. 57,PP.270-278,1975.

[19] V.S.Mosca, Calcaneal lengthening for valgus deformity of the hindfoot. Results in children who had severe, symptomatic flatfoot and skewfoot. J Bone Joint Surg.VOL.77,PP.500512,1995 .

[20] K.Rome, R.L.Ashford, A.Evans, Non-surgical interventions for paediatric pes planus. Cochrane Database Syst Rev.VOL.(7),PP. 1002-6311,2010. 